\title{
Las Revistas Científicas en Comunicación \\ Principales Retos \\ Iberoamericanos Actuales
}

https://doi.org/10.21814/uminho.ed.43.8

\section{Enrique Bustamante ${ }^{1}$}

Departamento de Ciencias de la Comunicación, Facultad de Ciencias de la Información, Universidad Complutense de Madrid, España

\footnotetext{
${ }_{1}^{1}$ In memorian.... Texto escrito e revisto pelo autor em 2020.
} 



\section{Introducción}

Las revistas científicas de Comunicación asumen hoy una trascendencia especial en las Ciencias Sociales, al superar con mucho el campo del periodismo y abarcar una visión transversal que atraviesa todas las actividades sociales y económicas, pero esa función de encrucijada trae consigo también enormes riesgos de atomización y dispersión temática.

Tales caracteres acentúan en la Comunicación los peligros actuales de las publicaciones en Ciencias Sociales: la colonización del Inglés empobrece la investigación y sus resultados, la hegemonía de los índices anglosajones margina las aportaciones iberoamericanas y latinas y las subordina a dinámicas comerciales de grandes grupos editoriales, la dominación de los grandes grupos multimedia anglosajones y su distribución en grandes paquetes arrincona aún más la economía y el impacto de la investigación latinoamericana en un escenario cada vez más global; unas tendencias peligrosas que las políticas científicas nacionales, medrosas y subordinadas, de los países latinos no han hecho más que aumentar, hasta delegar a veces su soberanía científica en el mundo angloamericano.

Mi experiencia sobre la prolongada historia de 32 años de Telos (1985-2017) de la que fui fundador y director hasta su conversión en revista de divulgación tecnológica y genérica, una de las publicaciones más veteranas en el ámbito español, me permitió plantearme durante años las grandes disyuntivas posibles: orientación académica o también profesional, investigadores emergentes o seniors, protocolos informáticos o atención directa a los autores, profesionalización de la edición o de la distribución, papel o soporte digital, todo pago o acceso abierto... Pero he comprobado, en diversos foros y debates, como en el coloquio organizado con motivo del $30^{\circ}$ aniversario de la revista Réseaux, en enero de 2014, que los problemas que he vivido en primer plano se pueden aplicar a prácticamente toda la Europa continental.

El cuadro conjunto de situación apela inevitablemente a la necesidad urgente de una cooperación iberoamericana acrecentada, que debería engarzarse en una cooperación latina creciente, tanto en la creación de índices prestigiosos autóctonos, como en la constitución de redes de distribución y promoción conjuntas y, finalmente, en la integración de las revistas científicas en Comunicación en una auténtica política de apoyo a la investigación en Ciencias Sociales.

\section{Comunicación: revista disciplinaria o temática}

Esa función de cruce de caminos trae consigo también enormes riesgos de generalización (ver bosques sin árboles) y de dispersión temática (árboles sin bosques). 
Ciertamente, se trata de reivindicar la Comunicación como un objeto de estudio axial en nuestra sociedad que ha pasado en varias décadas de una concepción restrictiva, los medios de comunicación de masa, a situarse en el centro del funcionamiento y del desarrollo de las sociedades contemporáneas; Comunicación también como encrucijada de caminos disciplinares múltiples, que se enriquece cada día con las aportaciones realizadas desde las perspectivas diversas de las Ciencias Sociales.

Pero esta concepción de la Comunicación exige asimismo una ambición totalizadora de su realidad social, conseguida a través de múltiples investigaciones empíricas, pero capaz de no perder de vista la complejidad global de la evolución de la comunicación social sobre la sociedad entera; una perspectiva que permite mantener la capacidad trascendental de crítica de la realidad, y su corolario imprescindible de interrogantes sobre cómo podría ser la Comunicación - y la sociedad - de otra forma, por otras vías, en beneficio de la mayoría de sus ciudadanos.

Los cantos a la delimitación superespecializada de cada revista de Comunicación, argumentados casi siempre en su probabilidad de aumentar las citas y los índices de impacto, corren el riesgo pues de incrementar la segmentación - disciplinar, sectorial o tecnológica -, reduciendo drásticamente esa capacidad holística de contemplar las relaciones entre comunicación y sociedad, en donde los departamentos estancos son cada vez más reducidos y pequeños. $Y$ por las mismas razones una revista que ofrezca sólo dossiers monográficos en cada número, satisfaciendo a una parte seguramente minoritaria de su público mientras frustra al resto, perdería su capacidad de cita periódica y estable con su audiencia.

\section{Revistas anglófonas o en lenguas latinas}

La pregunta es enormemente trascendental en este período del desarrollo del pensamiento en los países no anglófonos, porque implica consecuencias graves no sólo para las revistas científicas sino también para la investigación en Comunicación entera. Primero, porque como se sabe, la lengua implica también estructuras de pensamiento, conceptos, tradiciones, escuelas, riqueza de perspectivas epistemológicas incluso, que se harían mucho más limitadas, sesgadas y pobres en un mundo uniforme en Inglés. Segundo, porque la imposición de la publicación hegemónica o casi total en Inglés beneficia claramente la competitividad científica de los países de esa área y perjudica notablemente, en términos de riqueza de expresión y de costes económicos incluso, a los investigadores de las restantes áreas lingüísticas. Tercero, resulta altamente dudoso que la simple versión exclusiva en Inglés permita penetrar realmente en el ambiente científico anglosajón, en tanto que distancia a las publicaciones de la audiencia de sus propias naciones de origen, acentuando la distancia entre las Ciencias Sociales y la realidad misma investigada respecto al tejido social de sus países. 
Otra cosa es la edición en Inglés - ¿por qué no también en otras lenguas mayoritarias como el Portugués, el Español, el Francés...? - de los resúmenes y palabras clave, como vía para un intercambio cultural y científico, como garantía de mantenimiento de la riqueza de la lengua originaria de cada artículo cuando esta es comprensible por la mayoría de la audiencia en cada país. En cuanto a la opción de la doble lengua sistemática de cada revista, en su lengua originaria y en Inglés, resulta evidentemente de un coste suplementario y muchas veces prohibitivo para su editorial o para sus autores (hay revistas españolas que obligan a pagar la traducción a alto precio para garantizar su calidad). Pero, además, supone una confesión de debilidad de una cultura que, difícilmente le permitirá un diálogo de igual a igual con las revistas y el mundo científico anglosajón.

\section{Los índices de calidad}

Dicho esto, hay que reconocer que la presión en muchas naciones para la publicación de los textos y la edición única en Inglés de las revistas resulta muchas veces insoportable como consecuencia del provincianismo de las autoridades educativas y científicas que, como en Portugal o en la propia España han dado absoluto y acrítico reconocimiento a los índices académicos anglosajones. Así, la web del Consejo Superior de Investigaciones Científicas (CSIC) (homólogo del Centre National de la Recherche Scientifique [CNRS] francés), ha llegado a conectar automáticamente con el sitio web del Institute for Scientific Information (ISI) de Thomson Reuters, sus catálogos y sus índices de impacto, en una absoluta cesión de la soberanía nacional científica. Y de la misma forma la Agencia Nacional de Evaluación de la Calidad y Acreditación (ANECA), agencia de calidad científica española, todopoderosa para la promoción y carrera profesional de los investigadores y profesores, sólo aceptaba durante años a las revistas y los índices del ISI (Web of Knowledge [WoK]) o los de Scopus (de Elsevier), relegando a valores marginales a las publicaciones más destacadas españolas o latinoamericanas.

El asunto es tanto más escandaloso por cuanto que se trata de dos aparatos dependientes de grandes grupos privados en los que los índices forman parte de sus modelos de negocio, ellos mismos implicados en la edición de revistas científicas, y que, a mi modo de ver, ofrecen resultados fuertemente sesgados a favor de las revistas anglosajonas (entre el $80-87 \%$ de sus listados), que aparecen desde hace años sobre representadas en sus catálogos de Ciencias Sociales y Humanidades, dominando absolutamente el famoso "primer cuartil" frente a un número ridículo de revistas de otras áreas; en el segundo cuartil, apenas hay entre una y tres publicaciones chinas, españolas, brasileñas o francesas, la mayoría de las cuales aparece a partir del puesto 140-150. 
Los tres índices hegemónicos, Journal Citation Reports (JCR), Scopus y Web of Science son "muy sesgados a favor del mundo anglófono" en todas las Ciencias Sociales, y dejaban a las publicaciones en Español, y en Portugués, Francés o Italiano, en posiciones marginales: en JCR, de 2.731 publicaciones contempladas (2010), solo un 0,97\% estaban en Español (81, de las cuales 47 de España y el resto de Latinoamérica) y 25 en Francés (pero 85 de Australia); más significativo era aún que el Español representaba el 1,1\% de los artículos indexados (Ramos-Torre \& Callejo-Gallego, 2013, p. 51). Por su parte, en Scopus, de 3.015 revistas analizadas (2012), un 3,6\% eran en Español (79 españolas y 42 en Latinoamérica; Ramos-Torre \& Callejo-Gallego, 2013, p. 51).

Felizmente, el índice Hirsch y su evaluación a través de Google Scholar Metrics ha venido a disminuir estas discriminaciones, desde 2012, "socializando" o "democratizando" los índices de calidad, pero no a eliminarlas. En sus listados de impacto, más exhaustivos que los de Elsevier o Thomson Reuters aparece en la última década "sólo" un 59,67\% de revistas publicadas en Inglés y procedentes de los países anglosajones, y un número mayor pues de revistas de otros países (de entre 664 de 30 países) aunque en posiciones muy retrasadas respecto a las anglosajonas. A título de ejemplo, Réseaux fue la primera europea continental en el índice $\mathrm{H}$, durante cinco años (2007-2011), pero aparecía en el puesto 98 del ranking (de 272 en Comunicación, con más de 100 artículos publicados en los últimos cinco años), mientras que Telos encabezaba la lista de revistas en Español, pero en el puesto 101; puestos mantenidos durante una década porque, en el ranking de una década (2001-2010), Réseaux era ya la primera revista francesa y europea continental por citas y Telos la primera del mundo de habla hispana.

En el ranking de 2014 (sobre cinco años anteriores, 2009-2013), de un total de 423 publicaciones consideradas de Comunicación, había cinco latinas entre las primeras 100, y 21 entre las primeras 200: la primera publicación latina (brasileña) estaba en el puesto 31 y la segunda (italiana), en el 33, mientras que la primera francesa ocupaba el puesto 89 (Réseaux). En lo que respecta a España, figuraban tres revistas entre las primeras 100, y cuatro en las 150 iniciales del ranking.

Ciertamente, en muchos países latinos, se ha hecho un gran esfuerzo de adaptación en los últimos años. Pero también se ha dado una enorme dispersión de criterios e indicadores, desde las condiciones formales de autonomía editorial a los protocolos burocráticos, desde los índices de impacto seguidistas del mundo anglosajón a las encuestas de expertos españoles a veces de extrañas distorsiones en la selección; y, en correspondencia, se ha producido una proliferación de organismos y termómetros nacionales e internacionales, que en el caso español producen ya una notable confusión cruzada: Difusión y Calidad Editorial de las Revistas Españolas de Humanidades y Ciencias Sociales y Jurídicas (DICE), organizada por el CSIC, financiada por 
la agencia de calidad universitaria ANECA como referencia de calidad de las publicaciones científicas; Fundación Española para la Ciencia y la Tecnología (FECYT), In-RECS (Índice de Impacto de Revistas Españolas de Ciencias Sociales)... Además de Latindex y los indicadores anglosajones, no pocas veces mezclados.

Así, la avalancha de criterios formales para las revistas, algunas justas para avanzar en su profesionalización y rigor, degenera sin embargo en muchos casos en condiciones irracionales, en rígidos protocolos metodológicos que encorsetan la reflexión (tomados generalmente de las Ciencias de la Naturaleza o de la Psicología Médica) o en incontables condiciones burocráticas que condicionan la libertad investigadora, sus objetos de estudio y sus resultados.

De esta forma, al amparo de supuestos labels de calidad, se imponen decenas de condiciones formalistas, se calcula a priori incluso el porcentaje de textos rechazados, o se cae en la picaresca de la "ingeniería" de las autocitas, no ya de los propios autores sino de la publicación editora que en algunas revistas incluye explícita o implícitamente la exigencia abusiva de citar múltiples veces a la revista deseada para engordar su supuesto impacto, a sabiendas de que el índice $\mathrm{H}$ no permite discriminar las citas rigurosas y motivadas de las autocitas de los autores, incluyendo las citas cruzadas y pactadas entre grupos, ni siquiera esas autocitas impuestas abusivamente y hasta insertadas unilateralmente por las propias publicaciones. Una práctica tan irregular y acientífica como los consejos que nos rodeaban a muchos doctorandos y profesores en los años 80: no dejar de citar nunca todos los catedráticos del área... Por si acaso "caían" en los tribunales.

En la misma línea de exigencias perniciosas, se exige el uso de plataformas informáticas anónimas cuyas cuantiosas condiciones formales (que a menudo sustituyen a la labor de edición) pueden determinar el rechazo previo de artículos durante largo tiempo (hasta un año en algunos casos) sin contacto posible alguno entre editor y colaborador. Se olvida también con frecuencia en esa dinámica la originalidad y calidad del pensamiento desarrollado, en beneficio de la cantidad de producción, o de un conocimiento de corta memoria (la de internet generalmente), repetitivo cuando no inútil y no acumulativo ni progresivo. Y hasta el impacto de cada texto frente a la comodidad del índice de impacto del soporte de edición.

\section{La disyuntiva papel - digital}

Ciertamente, lo importante de una revista científica es la calidad de los contenidos, pero el soporte no es una cuestión trivial. Y en los tiempos de transición en que estamos, mi respuesta para una publicación periódica de Comunicación es no disyuntiva: en papel y en digital, a ser posible en este último caso, gratuita y de libre acceso. 
El papel sigue teniendo funciones esenciales para las bibliotecas públicas, para los investigadores especializados, para muchos países en desarrollo: recuerdo haber contemplado con emoción en facultades de Cuba pero también de Colombia, de Argentina y Brasil colecciones de Telos desgastadas por el prolongado uso.

En cambio, el soporte digital tiene ventajas indudables de acceso universal, de traspaso de las fronteras y las tasas aduaneras, tan abundantes en Iberoamérica, de conexión en todo tiempo y lugar, de descenso de costes. En teoría también de espacio ilimitado para muchos más artículos, pero esta posibilidad resulta inviable cuando los índices miden las citas en función del número de artículos editados, porque se rebajaría inmediatamente el impacto de una revista que duplicara el número de textos. Además, lo digital permite la proliferación y conexión de las bases de datos, el funcionamiento de los buscadores, la generación de espacios de debate, las dobles versiones lingüísticas, el enlace con las redes sociales y el consiguiente feedback con los lectores.

Los interrogantes más fuertes no residen pues en el soporte, sino en los modelos de negocio: difícil venta por número o suscripción digital, salvo en el caso de revistas anglosajonas que se agrupan en redes manejadas por potentes grupos editores para hacer pagar abonos excesivos (seis editoriales anglos editan en 2013 entre el 54$65 \%$ de las revistas de los rankings de WoK o Scopus). Difícil ecuación, salvo en caso de patrocinio privado, cuando nuestras universidades públicas y grupos de investigación están siendo esquilmados presupuestariamente por los estados en Europa y, especialmente, en España, mientras que las opciones de venta o suscripción por revista aislada se revelan inviables.

Tan grave o más que la cuestión financiera son las consecuencias que los índices están teniendo sobre el mundo científico completo de las Ciencias Sociales, en analógico o en digital. Así, la generalización de las valoraciones de doble ciego está alejando a los investigadores seniors reconocidos de las revistas, y dejando a estas como soporte casi único para los autores emergentes, incluidos los especialistas en promoción de carreras universitarias ("investigadores del boletín oficial del estado"), incluyendo frecuentemente exigencias formales irracionales, como la tasa mínima del $60 \%$ de rechazo de originales. Se está generando así un doble circuito perverso, con dos mundos paralelos sin casi conexión: los seniors publican libros, los jóvenes publican artículos en busca obsesiva de las "estrellas Michelin". Sólo los grandes congresos científicos periódicos se mantienen como lugar de encuentro, aunque penalizados incomprensiblemente cada vez más por las agencias de calidad de la enseñanza y la investigación, disminuyendo así tendencialmente el imprescindible diálogo entre investigadores nacionales e internacionales.

Para mayor abundamiento, el tejido editorial de libros, muy mayoritariamente privado y comercial, es mucho más fuerte y está más extendido en toda el área 
iberoamericana, con el detalle de que se calcula que un tercio de los libros de ensayo e investigación corresponden a las Ciencias Sociales en ese mercado; y ostentan índices de citas muchos más elevadas en su conjunto que las revistas. El problema es que la naturaleza comercial de esas editoriales resulta difícilmente compatible con las exigencias académicas: consejos científicos independientes de prestigio, evaluación anónima de originales, contabilización sistemática de los indicadores de impacto sobre obras cerradas y sujetas a copyright, entre otros. Las clasificaciones académicas de las editoriales y sus colecciones se hacen así frecuentemente subjetivas, sobre encuestas a expertos elegidos aleatoriamente o por su militantismo digital, y son despreciadas o marginalizadas en la evaluación de méritos de profesores e investigadores. De forma que la herramienta esencial de la historia para hacer avanzar el conocimiento social en profundidad se ve crecientemente relegada en la comunicación científica.

En fin y según un informe de 2013, las publicaciones (periódicas y monográficas) sobre Ciencias Sociales en el mundo hispanoamericano tenían unas "dimensiones acordes con la extensión y el dinamismo de las bases sociodemográficas del Español en el mundo"; y podríamos ampliar esta consideración al Portugués-Brasileño, al Francés e incluso al Italiano (Ramos-Torre \& Callejo-Gallego, 2013, p. 67). Pero esta "buena salud" del Español en el sistema científico de las Ciencias Sociales no quitaba su "escasa presencia en el sistema mundial de comunicación científica" (Ramos-Torre \& Callejo-Gallego, 2013, p. 67).

\section{Mi bagaje personal: la experiencia de 32 años de dirección de Telos}

No podría concluir este capítulo si no citara brevemente mi "bagaje personal" en el campo de las publicaciones sobre Comunicación, es decir aquellas experiencias que han determinado mis ideas en este campo: en primer lugar, inevitablemente, la revista Telos que fundé en 1985; en segundo término, la revista de la Asociación Española de Investigación de la Comunicación (AE-IC), RAEIC, Revista de la Asociación Española de Investigación de la Comunicación, que he contribuido a crear desde la directiva de la asociación.

\section{Telos}

Estoy obligado a referirme a la revista que fundé, en 1985, y he dirigido o coordinado hasta su reconversión en revista de divulgación tecnológica y que se ha convertido ya, 107 números y 32 años después (con un paréntesis de carencia de tres años - 1997-2001) en una de las decanas de la historia española junto con Análisis en España, o con Réseaux y con Media Culture \& Society en el Reino Unido. 
Editada originalmente por la Fundación de Desarrollo Comunal (Fundesco), que jugó un papel vital en la investigación sobre las nuevas tecnologías de información y comunicación (NTIC) hasta su desaparición, en 1997, Telos reapareció, en 2002, editada por la Fundación Telefónica, en una segunda etapa hasta 2007, aplicando ya de forma pionera las evaluaciones anónimas y los consejos científicos y de redacción independientes de la firma editora, pero amplió su temática al campo de las NTIC desde 2008, ajustándose a las condiciones de los índices internacionales. Desde 2009, especialmente, con el antetítulo de Revista de Pensamiento sobre Comunicación, Tecnología y Sociedad ha emprendido una gran transformación en red, y en formatos para tablets, consolidando su papel de publicación de referencia para el mundo iberoamericano.

En esta larga trayectoria de hemeroteca, con más de 2.000 autores publicados, entre ellos decenas de primeras figuras latinoamericanas, Telos ha sido ya objeto de investigaciones sobre el pensamiento comunicativo español e iberoamericano, que refleja, entre ellos de un destacado historiador que concluía que: "Telos consiguió en sus primeros 25 años (...) ser un referente del pensamiento comunicacional sobre las tecnologías y sobre la implantación de la sociedad de la información"; y añadía que la revista "desde sus inicios, nos permite seguir a través de sus páginas todo el debate académico, científico y tecnológico que ha rodeado el proceso de desarrollo de la sociedad de la información en el ámbito nacional e internacional" (Galindo, 2013, p. 134).

Pues bien, debo confesar que he sufrido en Telos todas las disyuntivas y los desgarramientos de un editor: comunicación general o especializada, papel o digital, Español o Inglés, acceso abierto (generalizado en Telos) o pago (en papel por venta o suscripción) resuelto gracias al generoso patrocinio de la Fundación Telefónica... Pero, sobre todo, he tenido que tomar opciones dolorosas en su orientación respecto a las exigencias de los índices: así, mantuvimos siempre la colaboración permanente de profesionales y expertos en Comunicación, lo que nos penalizaba frente a una visión exclusiva de académicos; hemos abierto espacios a los seniors en secciones de "autor invitado", lo que significaba también textos no evaluados anónimamente; hemos practicado una relación directa con los autores en cuanto a sus evaluaciones y modificaciones; hemos mantenido secciones amplias de actualidad e información para investigadores (regulación, investigaciones, agenda de congresos); hemos priorizado el diálogo entre investigación española e internacional y, dentro de esta, la de origen latinoamericano y europea; hemos resistido con los textos en Español (con títulos y resúmenes en Inglés), aun duplicando los artículos en su idioma original en la web, y a sabiendas de que seríamos castigados por los índices anglos... 


\section{RAE-IC}

Se trata de la revista oficial de la AE-IC (Asociación Española de Investigación de la Comunicación) que presido, confiada a un equipo de investigadores de la Universidad de Santiago de Compostela, dirigido por el profesor Xose López, aunque con total autonomía de funcionamiento: las secciones temáticas proponen temáticas de dossiers pero la evaluación es completamente independiente y anónima. Editada, desde principios de 2014, en soporte digital y con vocación internacional, ha conseguido ya en sus 14 números publicados, a finales de 2020, una notable diversidad de autores y temas, especialmente como promoción de los jóvenes investigadores y está entrando en los índices nacionales e internacionales.

Pero el camino seguido no ha sido fácil porque los autores buscan ansiosamente revistas ya consagradas en los índices y hacen falta años de esfuerzo financiero e intelectual para poder situarse entre las revistas de impacto nacional e internacional.

\section{La cooperación iberoamericana}

En conclusión de las reflexiones anteriores sobre la difusión de la investigación y las publicaciones científicas, podemos avanzar las siguientes sugerencias:

- $\quad$ precisamos de criterios estables y equilibrados de evaluación del trabajo investigador, articulados entre revistas periódicas, libros y sobre todo monografías, y grandes congresos internacionales de probada selección independiente, para que compongan un universo armónico capaz de proyectar la realidad de la investigación de cada universidad, región, país y autor;

- $\quad$ el mundo latino precisa con urgencia construir sus propios índices internacionales, en papel y en digital, que garanticen condiciones formales básicas de autonomía editorial y de rigor científico, pero que se centren también en el examen de la originalidad, de la calidad de la investigación, de la trascendencia de los objetos de estudio;

- tenemos que construir labels de prestigio por consenso, comunes al menos en las tres lenguas latinas de mayor difusión mundial (Español, Portugués, Francés), un nuevo Latindex que abarque tanto las condiciones formales de rigor y autonomía editorial como el impacto real de cada una en el mundo, un índice único que realmente funcione y sea compartido por las publicaciones y por su ambiente científico, y que sume además la voluntad de erradicar las malas prácticas y la competitividad mercantil descarnada; 
- necesitamos avanzar en la profesionalización de la edición, pero sin despegar esa tarea de la experiencia investigadora; afirmar el compromiso con la objetividad de la evaluación y el rigor de la reflexión, pero sin aislar a los seniors de las publicaciones periódicas de prestigio ni a los juniors de las ediciones monográficas; y progresar en la lucha contra la endogamia universitaria, pero sin romper los puentes con el mundo de la investigación profesional. Todo un arsenal de delicados equilibrios que es preciso calibrar finamente para fomentar la auténtica investigación de calidad;

- tenemos que avanzar en la construcción de redes de revistas - y de editoriales - públicas y privadas en el ámbito iberoamericano, pero también latino (de forma cruzada, entrelazada y mutua) capaz de promocionar y difundir solidariamente a escala internacional nuestros call for papers, nuestras ediciones y aportaciones en Comunicación, de internacionalizar cooperativamente nuestra forma de pensar y entender el mundo;

- Las revistas científicas académicas o no en Comunicación, deben estar orientadas a la difusión y transferencia de los avances de la investigación hacia la sociedad y no sólo ni fundamentalmente, como ocurre ahora, hacia el mundo académico, en un bucle autista - la lectura y cita mutua - que amenaza cortar nuestros vínculos con la sociedad, máxima finalidad de nuestra labor. Lo que implica también una reorientación de las temáticas y los objetos de estudio en función de las demandas y los intereses sociales mayoritarios, y de unas metodologías y unas conclusiones comprometidas con ellos.

En definitiva, tenemos que repensar y reformar profundamente las políticas públicas en la Ciencia en nuestros países en cuanto a las Ciencias Sociales en general, a la Comunicación en particular. Poniendo como foco de nuestro trabajo, de investigación y difusión, los grandes interrogantes que repetía incansablemente el gran intelectual que fue Héctor Schmucler: investigar ¿para qué? ¿Y para quién?

\section{Referencias}

Galindo, J. A. G. (2013). Telos, una revista científica sobre la sociedad de la información (1985-2009). Perspectivas de la Comunicación, 6(11), 134-158. http://ojs.ufro.cl/index.php/perspectivas/article/viewFile/154/131

Ramos-Torre, R. ,\& Callejo-Gallego, J. (2013). El Español en las ciencias sociales. In J. L. García, J. A. Alonso \& J. C. Jiménez (Eds.), El Español, lengua de comunicación científica (pp. 29-74). Fundación Telefónica; Ariel. 